\title{
Deforming charged black holes with dipolar differential rotation boundary
}

\author{
Tong-Tong $\mathrm{Hu}^{\mathrm{a}}$, Shuo Sun ${ }^{\mathrm{b}}$, Hong-Bo Lic ${ }^{\mathrm{c}}$, Yong-Qiang Wang ${ }^{\mathrm{d}}$ \\ Research Center of Gravitation, Institute of Theoretical Physics, Key Laboratory for Magnetism and Magnetic of the Ministry of Education, \\ Lanzhou University, Lanzhou 730000, China
}

Received: 5 December 2019 / Accepted: 15 June 2020 / Published online: 2 July 2020

(C) The Author(s) 2020

\begin{abstract}
Motivated by the recent studies of the novel asymptotically global $\mathrm{AdS}_{4}$ black hole with deformed horizon, we consider the action of Einstein-Maxwell gravity in AdS spacetime and construct the charged deforming AdS black holes with differential boundary. In contrast to deforming black hole without charge, there exists at least one value of horizon for an arbitrary temperature. The extremum of temperature is determined by charge $q$ and divides the range of temperature into several parts. Moreover, we use an isometric embedding in the three-dimensional space to investigate the horizon geometry. The entropy and quasinormal modes of deforming charged AdS black hole are also studied in this paper. Due to the existence of charge $q$, the phase diagram of entropy is more complicated. We consider two cases of solutions: (1) fixing the chemical potential $\mu$; (2) changing the value of $\mu$ according to the values of horizon radius and charge. In the first case, it is interesting to find there exist two families of black hole solutions with different horizon radii for a fixed temperature, but these two black holes have same horizon geometry and entropy. The second case ensures that deforming charged AdS black hole solutions can reduce to standard RN-AdS black holes.
\end{abstract}

\section{Contents}

1 Introduction . . . . . . . . . . . 1

2 Action and numerical method . . . . . . . . 2

3 Numerical model . . . . . . . . . . . . . . . . . . 3

4 Black hole solutions with fixed value of $\mu \ldots \ldots 5$

4.1 Horizon geometry ................ 5

4.2 Entropy . . . . . . . . . . . 7

\footnotetext{
a e-mail: hutt17@1zu.edu.cn

be-mail:sunsh17@1zu.edu.cn

c e-mail: lihb2017@1zu.edu.cn

de-mail: yqwang@lzu.edu.cn (corresponding author)
}

4.3 Stability ................... . . 9

5 Black hole solutions with $\mu=\frac{2 q}{y_{+}} \ldots \ldots . . .10$

6 Conclusions and outlook . . . . . . . . . . 11

References . . . . . . . . . . . . . . . 12

\section{Introduction}

In classical general relativity, due to the uniqueness theorem of black holes [1-4], the asymptotically flat charged black hole solutions with zero angular momentum in four dimensions are named as Reissner-Nordstrom (RN) black holes, which have two spherical event horizons. In four-dimensional anti-de Sitter (AdS) spacetime, it is well-known that except for compact horizons of arbitrary genus, there exist some solutions with noncompact planar or hyperbolic horizons. Because of the development of Anti-de Sitter/conformal field theory (AdS/CFT) correspondence [5-7], it becomes more important to study physical properties of AdS black holes.

Because the asymptotically AdS black hole has a boundary metric of conformal structure, we could deform the boundary metric to obtain a family of solutions of black hole with deformed horizon, whose curvature is not a constant value. There are many works in this field with both analytical and numerical methods. For the analytic method, the authors in [8] constructed a family of black hole solutions with deformed horizons in four-dimensional spacetime by using AdS C-metric [9-11]. In addition, a class of solutions of four-dimensional AdS black holes with noncompact event horizons of finite area was found in [12,13], and black holes with bottle-shaped event horizon were founded analytically in [14]. With numerical methods, the authors in [15] got a family of deforming solutions including soliton and black hole with dipolar differential boundary $\Omega(\theta)=\varepsilon \cos (\theta)$. The constant $\varepsilon$ is the boundary rotation parameter and $\theta$ is polar angle. When $\varepsilon>2$, the norm Killing vector $\partial_{t}$ becomes 
spacelike for certain regions which also are called as ergoregions, and deforming AdS black holes with ergoregions may be unstable due to superradiant scattering [16]. Because of superradiance, both solitons and black holes develop hair at $\varepsilon>2$. Motivated by this work, we also studied deforming solutions with odd multipolar [17] and even multipolar [18] differential rotation boundary. Furthermore, the authors in [19] numerically studied a class of vacuum solutions with a noncompact, differential rotation boundary metric. With AdS C-metric, the effect of changing boundary metric on hyperbolic and compact AdS black holes had been studied in [20]. Considering the matter fields, the authors in [21] constructed the deforming black holes in $D=5$ minimal gauged supergravity. Moreover, the authors in $[22,23]$ constructed new asymptotically locally $A d S_{5}$ black holes with squashed boundary in $N=2, D=5$ Fayet-Iliopoulos supergravity theory, which generalized the minimal gauged supergravity solutions to the Fayet-Iliopoulos theory with non trivial running scalars in the supersymmetric case.

Until now, the works of deforming AdS black hole with differential boundary $[15,17,18]$ were only studied in the cases without charge. It would be interesting to see whether there exist the charged deforming AdS black hole solutions in Einstein-Maxwell-AdS spacetime. In this paper, we would like to numerically solve coupled Einstein-Maxwell equations to obtain a family of solutions of charged deforming black holes. These solutions have the anti-symmetric rotation profile on the equatorial plane, which keeps total angular momentum of black hole being zero. In contrast to the cases without charge, there exist some new properties of black holes due to the existence of charge $q$. Firstly, there exists at least one value of horizon for an arbitrary temperature, while there exists no horizon when $T<T_{\min }$ for $q=0$. Besides, the extremum of temperature is determined by the charge $q$ and divide temperature into several parts according to the the values of charge $q$. In different regions of temperature, the number of values for horizon is different. Specifically, in the region with one value of horizon for a fixed temperature, there exist two families of solutions with same horizon when temperature is lower than the minimal extremum of temperature for RN-AdS black hole $T_{R N}=\frac{\sqrt{6}}{3 \pi}$. Furthermore, when we fix the value of chemical potential $\mu$, in the region with three values of horizon for a fixed temperature, it is interesting to find that two small branches have same properties such as horizon geometry, entropy and stability, although their horizon radii are not equal. If we require the deforming charged AdS black hole solutions to reduce to standard RNAdS black holes with boundary rotation parameter $\varepsilon=0$, the black holes with different horizon radii have different horizon geometry and entropy.

The plan of our work is as follows. In the next section, we introduce the action and numerical method. In Sect. 3, we give the numerical model and discuss the effect of the temperature $T$ and the charge $q$ on solutions. In Sect. 4, we fix the chemical potential $\mu$ and obtain numerical solutions of charged AdS black hole with differential rotation boundary, and show more properties of deforming charged AdS black hole including horizon geometry, entropy and stability. In Sect. 5, we give an example to show the numerical results of deforming charged AdS black hole which can reduce to standard RN-AdS black hole. The conclusions and outlook are given in the last section.

\section{Action and numerical method}

We start with Einstein-Maxwell action in four-dimensional AdS spacetime, whose action is given by

$S=\frac{1}{16 \pi G} \int \mathrm{d}^{4} x \sqrt{-g}\left(R-2 \Lambda-\frac{1}{4} F^{\mu \nu} F_{\mu \nu}\right)$,

where $G$ is the gravitational constant, $\Lambda$ is cosmological constant represented by AdS radius $L$ as $-3 / L^{2}$ in fourdimensional spacetime, $g$ is determinant of metric and $R$ is Ricci scalar.

The equations of motion of the Einstein and Maxwell fields which can be derived from the Lagrangian density (2.1) are as follows

$R_{\mu \nu}+\frac{3}{L^{2}} g_{\mu \nu}-\left(\frac{1}{2} F_{\mu \lambda} F_{\nu}{ }^{\lambda}-\frac{1}{8} g_{\mu \nu} F_{\lambda \rho} F^{\lambda \rho}\right)=0$,

$\nabla_{\mu} F^{\mu \nu}=0$.

The spherically symmetric solution of motion equations (2.2) is the well-known Reissner-Nordstrom-AdS ( RNAdS) black hole. The metric of RN-AdS black hole solution could be written as follows

$$
\begin{aligned}
d s^{2}= & -\left(1-\frac{2 M}{r}+\frac{q^{2}}{r^{2}}+\frac{r^{2}}{L^{2}}\right) d t^{2} \\
& +\left(1-\frac{2 M}{r}+\frac{q^{2}}{r^{2}}+\frac{r^{2}}{L^{2}}\right)^{-1} d r^{2}+r^{2} d \Omega^{2}
\end{aligned}
$$

and the gauge field is written as

$F=d A, \quad A=\frac{2 q}{r} d t$.

Here, $d \Omega^{2}$ represents the standard element on $S^{2}$, the constant $M$ is the mass of black hole measured from the infinite boundary, and the constant $q$ is the charge of black hole. The black hole mass is related to the charge $q$ and horizon radius $r_{+}$by the relation

$M=\frac{1}{2}\left(r_{+}+\frac{q^{2}}{r_{+}}+\frac{r_{+}^{3}}{L^{2}}\right)$, 
where $r_{+}$is the largest root. The Hawking temperature $T_{H}$ of $\mathrm{RN}-\mathrm{AdS}$ black hole is given by

$T_{H}=\frac{1}{4 \pi r_{+}}\left(1+\frac{3 r_{+}^{2}}{L^{2}}-\frac{q^{2}}{r_{+}^{2}}\right)$.

At near infinity the metric is asymptotic to the AdS spacetime, and boundary metric is given by

$d s_{\partial}^{2}=-d t^{2}+d \theta^{2}+\sin ^{2} \theta d \phi^{2}$.

In order to obtain the new asymptotic AdS solution, the authors in [15] add differential rotation to the boundary metric, which is given by

$d s_{\partial}^{2}=-d t^{2}+d \theta^{2}+\sin ^{2} \theta[d \phi+\Omega(\theta) d t]^{2}$,

with a dipolar differential rotation $\Omega(\theta)=\varepsilon \cos \theta$. The constant $\varepsilon$ is the amplitude of the boundary rotation. The norm of Killing vector $\partial_{t}$ is

$\|\partial t\|^{2}=-1+\frac{\varepsilon^{2}}{4} \sin ^{2}(2 \theta)$.

From the above equation, we could find that the maximal value of Killing vector appears at $\theta=\frac{\pi}{4}$. We will take the same dipolar differential rotation boundary (2.8).

In order to get a set of charged deforming black hole solutions, we would like to use DeTurck method [24-26] to solve equations of motion (2.2). By adding a gauge fixing term, we change equations (2.2a) to elliptic equations:

$$
\begin{aligned}
R_{\mu \nu} & +\frac{3}{L^{2}} g_{\mu \nu}-\left(\frac{1}{2} F_{\mu \lambda} F_{\nu}{ }^{\lambda}-\frac{1}{8} g_{\mu \nu} F_{\lambda \rho} F^{\lambda \rho}\right) \\
& -\nabla_{(\mu} \xi_{v)}=0
\end{aligned}
$$

where the DeTurck vector $\xi^{\mu}=g^{v \rho}\left(\Gamma_{\nu \rho}^{\mu}[g]-\Gamma_{v_{\rho}}^{\mu}[\tilde{g}]\right)$ is related to reference metric $\tilde{g}$. It is notable that the reference metric $\widetilde{g}$ should be chosen to have the same boundary and horizon structure with $g$. Using this method to solve equations (2.2b) and (2.10), we could obtain a family of charged AdS black hole solutions.

\section{Numerical model}

To obtain solutions of charged deforming AdS black hole, we start with this ansätze of metric,

$$
\begin{aligned}
\mathrm{d} s^{2}= & \frac{L^{2}}{\left(1-y^{2}\right)^{2}}\left\{-y^{2} \tilde{\Delta}(y) U_{1} \mathrm{~d} t^{2}+\frac{4 y_{+}^{2} U_{2} \mathrm{~d} y^{2}}{\tilde{\Delta}(y)}\right. \\
& +y_{+}^{2}\left[\frac{4 U_{3}}{2-x^{2}}\left(\mathrm{~d} x+x \sqrt{2-x^{2}} y U_{4} \mathrm{~d} y\right)^{2}\right. \\
& \left.\left.+\left(1-x^{2}\right)^{2} U_{5}\left(\mathrm{~d} \phi+y^{2} x \sqrt{2-x^{2}} U_{6} \mathrm{~d} t\right)^{2}\right]\right\},
\end{aligned}
$$

with

$$
\begin{aligned}
& \Delta(y)=\frac{-q^{2}\left(1-y^{2}\right)^{3}}{L^{2} y_{+}^{2}}+\left(1-y^{2}\right)^{2}+y_{+}^{2}\left(3-3 y^{2}+y^{4}\right), \\
& \text { and } \\
& \tilde{\Delta}(y)=\Delta(y) \delta+y_{+}^{2}(1-\delta),
\end{aligned}
$$

where the functions $U_{i}, i \in(1, \ldots, 6)$ depend on $x$ and $y$, the parameter $q$ is the charge of black hole, and $y_{+}$is horizon radius. Here, $y$ is related to radial coordinate $r$ with $r=L y_{+} /\left(1-y^{2}\right)$, and $x$ represents polar angle on $S^{2}$ with $\sin \theta=1-x^{2}$. When $U_{1}=U_{2}=U_{3}=U_{5}=\delta=1$ and $U_{4}=U_{6}=0$, the line element (3.1) can reduce to $\mathrm{RN}-\mathrm{AdS}$ black hole, and it is also the reference metric $\widetilde{g}$ for DeTurck method in this model.

Considering an axial symmetry system, we have polar angle reflection symmetry $\theta \rightarrow \pi-\theta$ on the equatorial plane, and thus it is convenient to consider the coordinate range $\theta \in[0, \pi / 2]$, i.e $x \in[0,1]$. We require the functions to satisfy the Neumann boundary conditions at $y=0$ and the equatorial plane $x=0$

$\partial_{y} U_{i}(x, 0)=\partial_{x} U_{i}(0, y)=0, \quad i=1,2,3,4,5,6$,

and set axis boundary conditions at $x=1$, where regularity must be imposed Dirichlet boundary conditions on $U_{4}$ and Neumann boundary conditions on the other functions

$U_{4}(1, y)=0$

and

$$
\begin{aligned}
\partial_{x} U_{1}(1, y) & =\partial_{x} U_{2}(1, y)=\partial_{x} U_{3}(1, y) \\
& =\partial_{x} U_{5}(1, y)=\partial_{x} U_{6}(1, y)=0 .
\end{aligned}
$$

At $y=1$, we set $U_{4}=0, U_{6}=\varepsilon$ and $U_{1}=U_{2}=U_{3}=$ $U_{5}=1$. Moreover, expanding the equations of motion near $x=1$ gives $U_{3}(1, y)=U_{5}(1, y)$.

In order to ensure that the number of unsolved functions is the same as that of equations in Deturck method, we introduce the component $A_{\phi}$ in gauge potential and choose the following form

$$
A=A_{t} d t+A_{\phi} d \phi
$$

where $A_{t}$ and $A_{\phi}$ are all real functions of $x$ and $y$. As for the boundary conditions of vector field, we set $A_{t}(x, 1)=\mu$ and $A_{x}(x, 1)=0$, where the constant $\mu$ is chemical potential which represents the asymptotic behavior of Maxwell field at infinity. At $x=1$, we choose $A_{t}(1, y)=0$ and $A_{x}(1, y)=0$.

The Hawking temperature of charged deforming black hole under ans $\ddot{a}$ tze (3.1) takes the following form:

$$
\begin{aligned}
T & =\left.\frac{1}{4 \pi} \sqrt{-g^{t t} g^{M N} \partial_{M} g_{t t} \partial_{N} g_{t t}}\right|_{r=r_{+}} \\
& =\frac{y_{+}^{4}+\delta\left(-q^{2}+y_{+}^{2}\left(1+2 y_{+}^{2}\right)\right)}{4 \pi y_{+}^{3}} .
\end{aligned}
$$


When the charge $q=0$, the formula (3.6) can reduce to the temperature of Schwarzschild-AdS black hole which was also given in [15]. For $\delta=1$, the extremums of temperature $T$ depend on the value of charge $q$ :

- $q=0$ : There is only a local minimum $T_{\min }=T_{S}=\frac{\sqrt{3}}{2 \pi}$, which is the minimal temperature of Schwarzschild-AdS black hole.

- $0<q<1 / 6$ : There are two extremums of temperature.

$$
\left\{\begin{array}{l}
T_{\min }=\frac{3 \sqrt{\frac{3}{2}}\left(-q^{2}+\frac{1}{12}\left(\sqrt{1-36 q^{2}}+1\right)^{2}+\frac{1}{6}\left(\sqrt{1-36 q^{2}}+1\right)\right)}{\pi\left(\sqrt{1-36 q^{2}}+1\right)^{3 / 2}}, \\
T_{\max }=\frac{3 \sqrt{\frac{3}{2}}\left(-q^{2}+\frac{1}{12}\left(1-\sqrt{1-36 q^{2}}\right)^{2}+\frac{1}{6}\left(1-\sqrt{1-36 q^{2}}\right)\right)}{\pi\left(1-\sqrt{1-36 q^{2}}\right)^{3 / 2}} .
\end{array}\right.
$$

- $q=1 / 6: T_{\max }=T_{\min }=T_{R N}=\frac{\sqrt{6}}{3 \pi}$, which is the minimal extremum of temperature for RN-AdS black hole.

- $q>1 / 6$ : There exists no extremum of temperature.

Next, we will analyze how the charge $q$ and temperature $T$ determine the number of values of horizon. In Fig. 1, we show the temperature $T$ as functions of horizon $y_{+}$at $\delta=1$ for several values of charge $q$. The black, blue, orange and green lines represent $q=0, \frac{1}{9}, \frac{1}{6}$ and $\frac{1}{4}$, respectively. For $q=\frac{1}{6}$, the intersection of the black horizontal and the vertical dashed lines indicates horizon $y_{+}=\frac{1}{\sqrt{6}}$ and $T_{R N}=\frac{\sqrt{6}}{3 \pi}$. For $q=0$, the intersection of the red horizontal and the vertical dashed lines indicates horizon $y_{+}=\frac{1}{\sqrt{3}}$ and $T_{S}=\frac{\sqrt{3}}{2 \pi}$. The number of values of horizon depends on different ranges of temperature $T$ and charge $q$ :

1. $q=0$ :

(a) $T<T_{S}$ : There exists no horizon.

(b) $T=T_{S}$ : There are two equal values of horizon $y_{+}=$

$\frac{1}{\sqrt{3}}$.

(c) $\stackrel{\sqrt{3}}{T}>T_{S}$ : There are two different values of horizon.

2. $0<q<1 / 6$ :

(a) $T<T_{\min }$ or $T>T_{\max }$ : There is one value of horizon.

(b) $T=T_{\min }$ or $T=T_{\max }$ : There are three values of horizon and two of them are equal.

(c) $T_{\min }<T<T_{\max }$ : There are three different values of horizon.

3. $q=1 / 6$ :
(a) $T=T_{R N}$ : There are three equal values of horizon $y_{+}=\frac{1}{\sqrt{6}}$.
(b) $T \neq T_{R N}$ : There is only one value of horizon.

4. $q>1 / 6$ : There is only one value of horizon.

In the following part of this section, we will discuss how these deforming charged AdS black hole solutions can reduce to standard RN-AdS black holes. As we mentioned above, for the line element (3.1), when $U_{1}=U_{2}=U_{3}=U_{5}=\delta=1$ and $U_{4}=U_{6}=0$, it can reduce to RN-AdS black hole as the following form.
Fig. 1 The temperature $T$ as functions of $y_{+}$for $\delta=1$. From top to bottom, the black, blue, red and green lines describe charge $q=0, \frac{1}{9}, \frac{1}{6}$ and $\frac{1}{4}$, respectively. The red and black horizontal dashed lines represent $T_{S}=\frac{\sqrt{3}}{2 \pi}$ and $T_{R N}=\frac{\sqrt{6}}{3 \pi}$. The red and black vertical lines represent $y_{+}=\frac{1}{\sqrt{3}}$ and $y_{+}=\frac{1}{\sqrt{6}}$

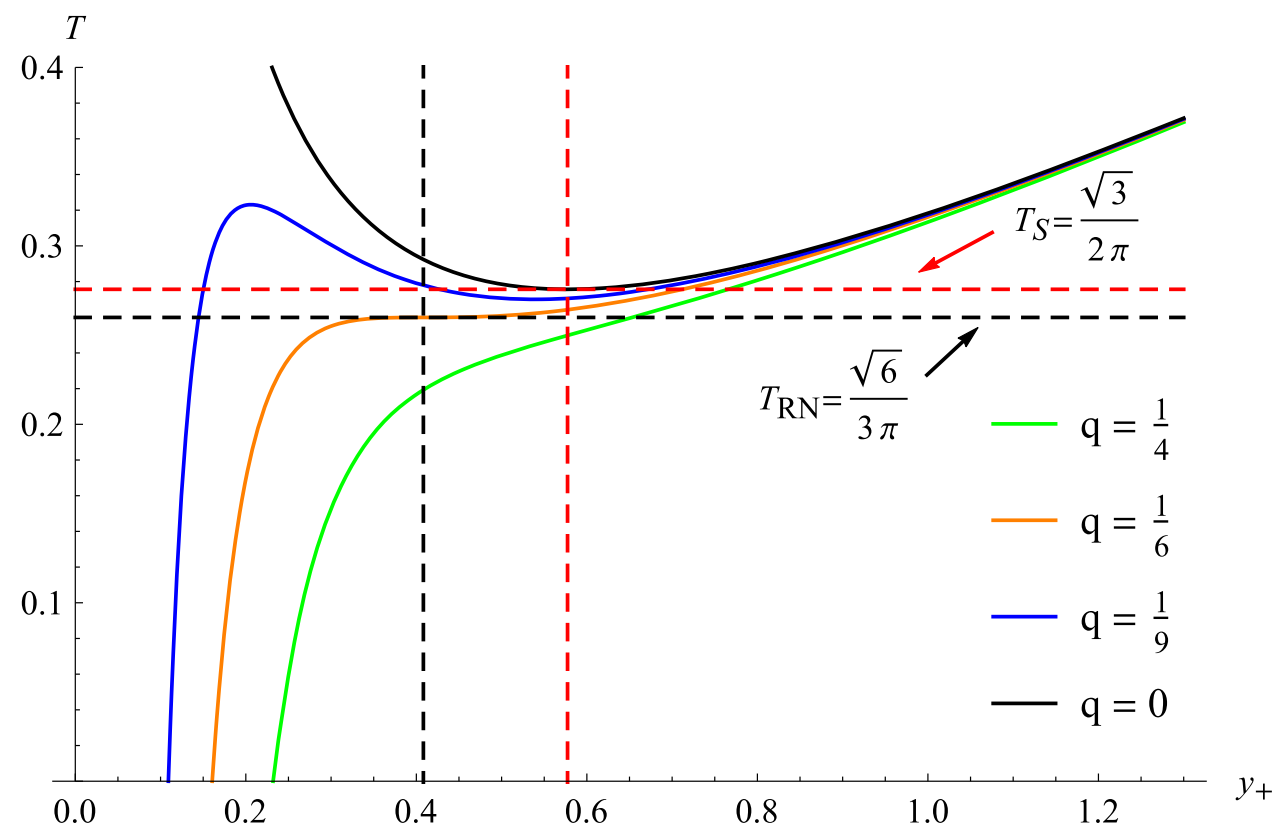




$$
\begin{aligned}
\mathrm{d} s^{2}= & \frac{L^{2}}{\left(1-y^{2}\right)^{2}}\left\{-y^{2} \Delta(y) \mathrm{d} t^{2}\right. \\
& +\frac{4 y_{+}^{2} \mathrm{~d} y^{2}}{\Delta(y)}+y_{+}^{2} \\
& \left.\times\left[\frac{4}{2-x^{2}} \mathrm{~d} x^{2}+\left(1-x^{2}\right)^{2} \mathrm{~d} \phi^{2}\right]\right\},
\end{aligned}
$$

with

$\Delta(y)=\frac{-q^{2}\left(1-y^{2}\right)^{3}}{L^{2} y_{+}^{2}}+\left(1-y^{2}\right)^{2}+y_{+}^{2}\left(3-3 y^{2}+y^{4}\right)$,

and the gauge filed component $A_{t}$ should be

$A_{t}=-\mu y^{2}$,

with $\mu=\frac{ \pm 2 q}{y_{+}}$. But when $\delta<1$, there exists no proportional relation between $\mu$ and $q$ when the boundary rotation parameter $\varepsilon=0$, and it means the deforming charged AdS black hole solutions for $\delta<1$ can not reduce to standard RN-AdS black hole. Actually, there exist coordinate transformations between this kind of non-standard RN-AdS black hole solutions and standard RN-AdS black holes. For convenience, we can fix the value of $\mu$ to obtain black hole solutions in this case.

By regulating parameter $\delta$, we can also get three values of horizon below the local minimal temperature $T_{\min }$. For simplify, we set the AdS radius $L=1$ in our numeral calculations.

We employ finite element methods in the integration region $0 \leq x \leq 1$ and $0 \leq y \leq 1$ defined on non-uniform grids, allowing the grids to be more finer grid points near the boundary of $y=1$. Our iterative process is the NewtonRaphson method. The relative error for the numerical solutions in this work is estimated to be below $10^{-5}$. In order to keep good agreement with the aforementioned error, the grid size has to be increased and typically a $120 \times 200$ to $120 \times 250$ grid was used.

\section{Black hole solutions with fixed value of $\mu$}

Considering there exists no proportional relation between $\mu$ and $q$ when $\delta<1$, we set $\mu=0.5$ in this section. There exist coordinate transformations between this kind of deforming charged AdS black hole solutions and standard RN-AdS black holes. We must point out that in this case, the black hole solutions with different horizon radii reduce to different non-standard RN-AdS black holes when $\varepsilon=0$. The numerical results can be seen as being obtained in different coordinate systems.

In Fig. 2, we give the plots of $U_{4}$ as functions of $x$ and $y$ for $T=0.42, \varepsilon=1.6$ and $\delta=1$. When fixing $q=$ $0.07057<\frac{1}{6}$, we can obtain three values of horizon. The distributions of $U_{4}$ for two small branches with $y_{+}=0.0992$ (left) and $y_{+}=0.1773$ (right) are given in the top of Fig. 2 . The left of bottom is the distributions of $U_{4}$ for large branch $y_{+}=1.5438$. To understand how the charge $q$ influences the function $U_{4}$, we also plot $U_{4}$ as functions of $y$ at the equatorial plane $x=1$ for several values of $q$. From top to bottom, the plots of function $U_{4}$ with charge $q=0,1.7068$, $2.2684,3.4299$ are represented by black, red, blue, green and pink lines, respectively. Due to the existence of relation (3.6), the horizon radius $y_{+}$increases with the increasing of charge $q$ for a fixed temperature.

\subsection{Horizon geometry}

In this subsection, we will study how the black hole horizon geometry behaves with the increase of boundary rotation parameters $\varepsilon$ and charge $q$. We could use an isometric embedding in the three-dimensional space [27-31] to investigate the horizon geometry of a two-dimensional surface in a curved space [15,32]. With the method provided by [15], the horizon of black hole is embedded into hyperbolic $H^{3}$ space in global coordinates:

$d s_{H^{3}}^{2}=\frac{d R^{2}}{1+R^{2} / l^{2}}+R^{2}\left[\frac{d X^{2}}{1-X^{2}}+\left(1-X^{2}\right) d \phi^{2}\right]$,

where $l$ is the radius of the hyperbolic space and we fix $l=0.73$ in our whole calculation. The induce metric of the horizon of black hole is the following form:

$d s_{H}^{2}=L^{2}\left[\frac{4 y_{+}^{2} U_{3}(x, 0)}{2-x^{2}} d x^{2}+y_{+}^{2}\left(1-x^{2}\right)^{2} U_{5}(x, 0) d \phi^{2}\right]$,

which can be obtained from the ansätze (3.1). The embedding is given by a curve with two parameters $\{R(x), X(x)\}$ and written by:

$$
\begin{aligned}
d s_{p b}^{2}= & {\left[\frac{R^{\prime}(x)^{2}}{1+\frac{R(x)^{2}}{l^{2}}}\right.} \\
& \left.+\frac{R(x)^{2} X^{\prime}(x)^{2}}{1-X(x)^{2}}\right] d x^{2}+R(x)^{2}\left(1-X(x)^{2}\right) d \phi^{2} .
\end{aligned}
$$

Equating this line element with induce metric (4.1.2), we can get the following first order differential equation:

$$
\begin{aligned}
0= & 4 H(x) P(x)\left(X(x)^{2}-1\right)^{2}\left[P(x)-l^{2}\left(X(x)^{2}-1\right)\right] \\
& +4 l^{2} P(x) X(x)\left(X(x)^{2}-1\right) P^{\prime}(x) X^{\prime}(x) \\
& -\left(X(x)^{2}-1\right)^{2} l^{2} P^{\prime}(x)^{2}-4 P(x)^{2}\left(l^{2}+P(x)\right) X^{\prime}(x)^{2}
\end{aligned}
$$



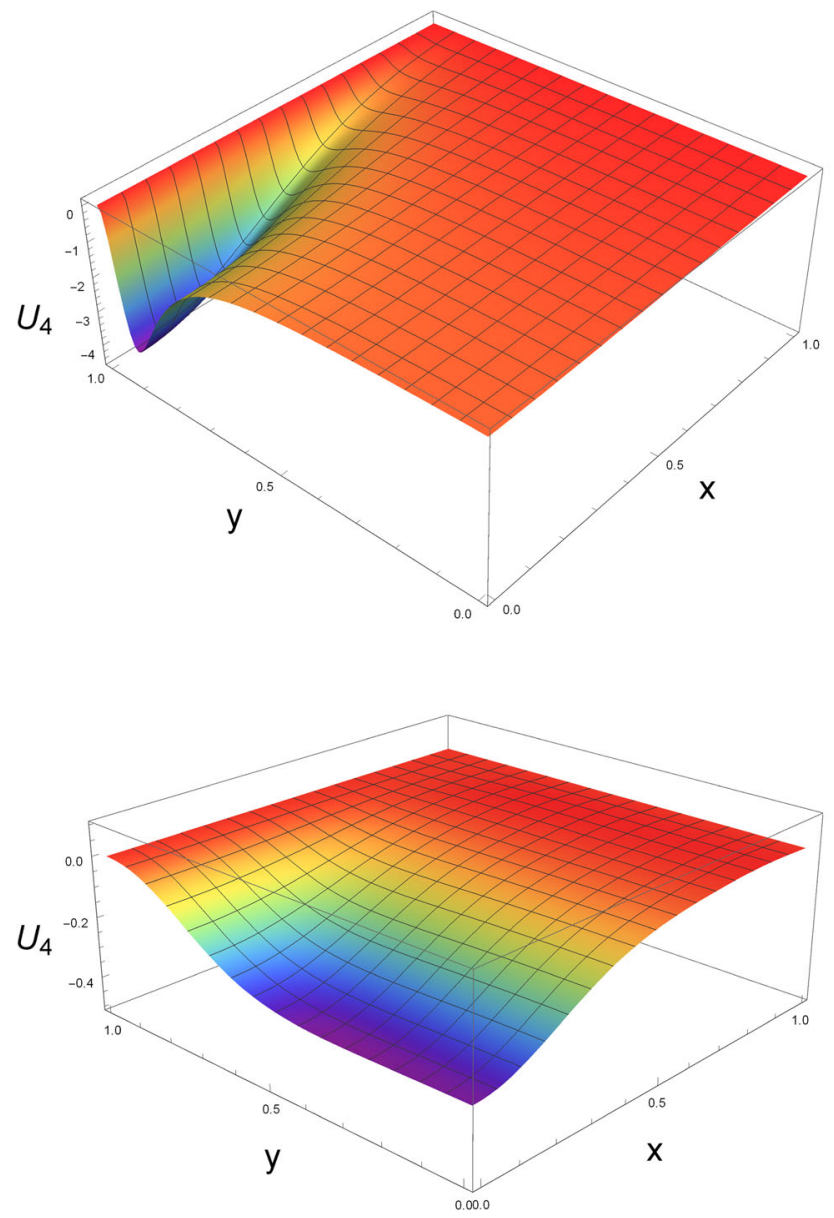

Fig. 2 Top: the plots of $U_{4}$ as functions of $x$ and $y$ of two small branches for $y_{+}=0.0992$ (left) and $y_{+}=0.1773$ (right). Bottom left: the plots of $U_{4}$ as functions of $x$ and $y$ for large branch $y_{+}=1.5438$. The three solutions of $U_{4}$ are given with $T=0.42, \varepsilon=1.6, \delta=1$ and $q=0.07057$. Bottom right: the plots of $U_{4}$ as functions of $y$ at the

where $H(x)=\left(2-x^{2}\right)^{-1}\left(4 y_{+}^{2} U_{3}(X, 0)\right)$ and $P(X)=$ $y_{+}^{2}\left(1-x^{2}\right)^{2} U_{5}(x, 0)$.

In Fig. 3, we show the hyperbolic embedding of the cross section of the large black hole horizon for different values of $\varepsilon$ with the charge $q=0.07057$ and the temperature $T=$ $0.42 \geq T_{\min }=0.2325$. From inner to outer, the black, red, green, orange and blue lines describe the boundary rotation parameter $\varepsilon=0.6,1.2,1.6,1.8$ and 1.9 , respectively. It is clear that the horizon deforms more dramatically with the increase of boundary rotation parameter $\varepsilon$.

Considering there is only one horizon radius when $T<$ $T_{\min }$ with $\delta=1$, we adjust $\delta<1$ to get three values of horizon and study the deformation of horizon for a fixed low temperature. In Fig. 4, we present hyperbolic embedding of the cross section of three black hole horizons for different values of $\varepsilon$ with $T=0.2585$ and $q=0.07057$. In the left panel, we show large black hole solutions for $y_{+}=0.9152$ and find that the size of the deformation of horizon cross sec-
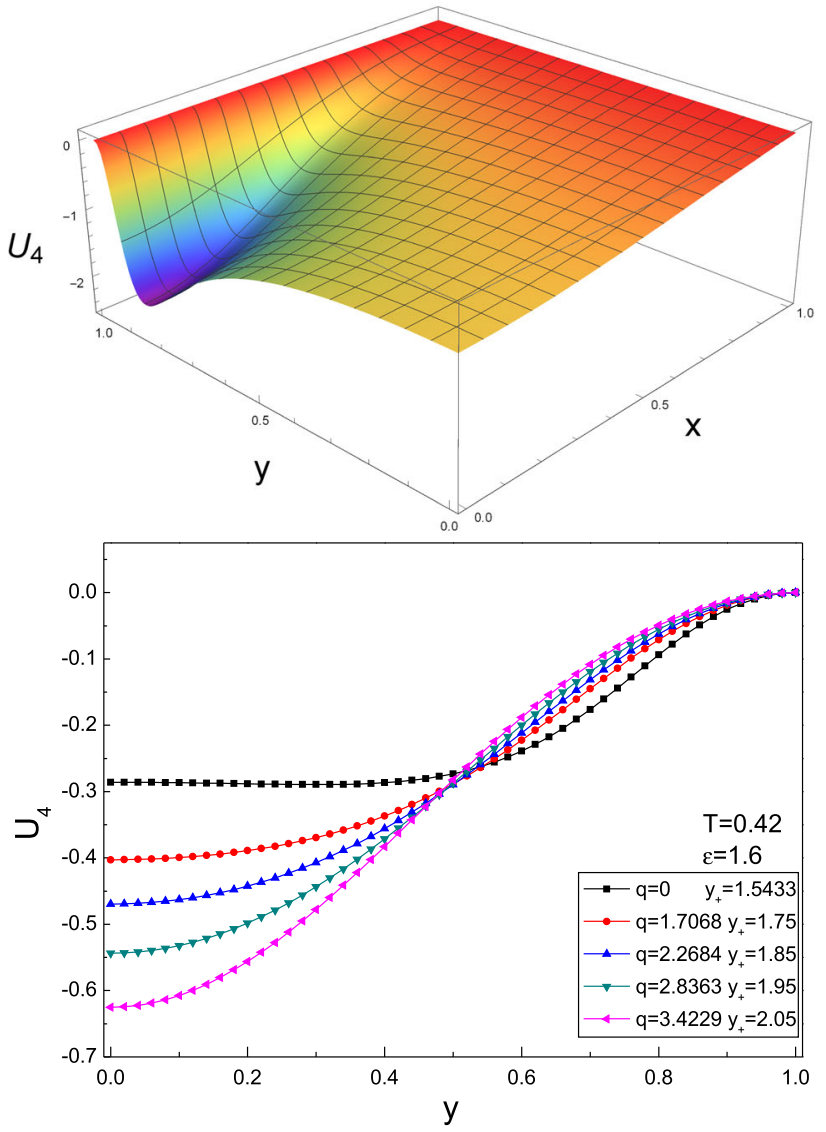

equatorial plane $x=1$ for several values of charge $q$ with $T=0.42$ and $\varepsilon=1.6$. From top to bottom, the black, red, blue, green and pink lines describe the charge $q=0,1.7068,2.2684,2.8363$ and 3.4299, respectively

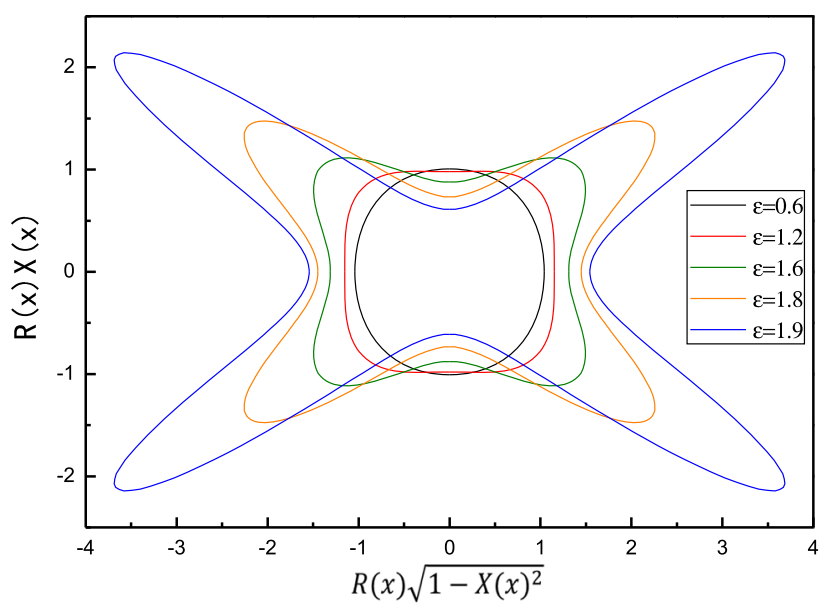

Fig. 3 Hyperbolic embedding of the cross section of the large black hole horizon for different values of $\varepsilon$ with $T=0.42$ and $q=0.07057$ 

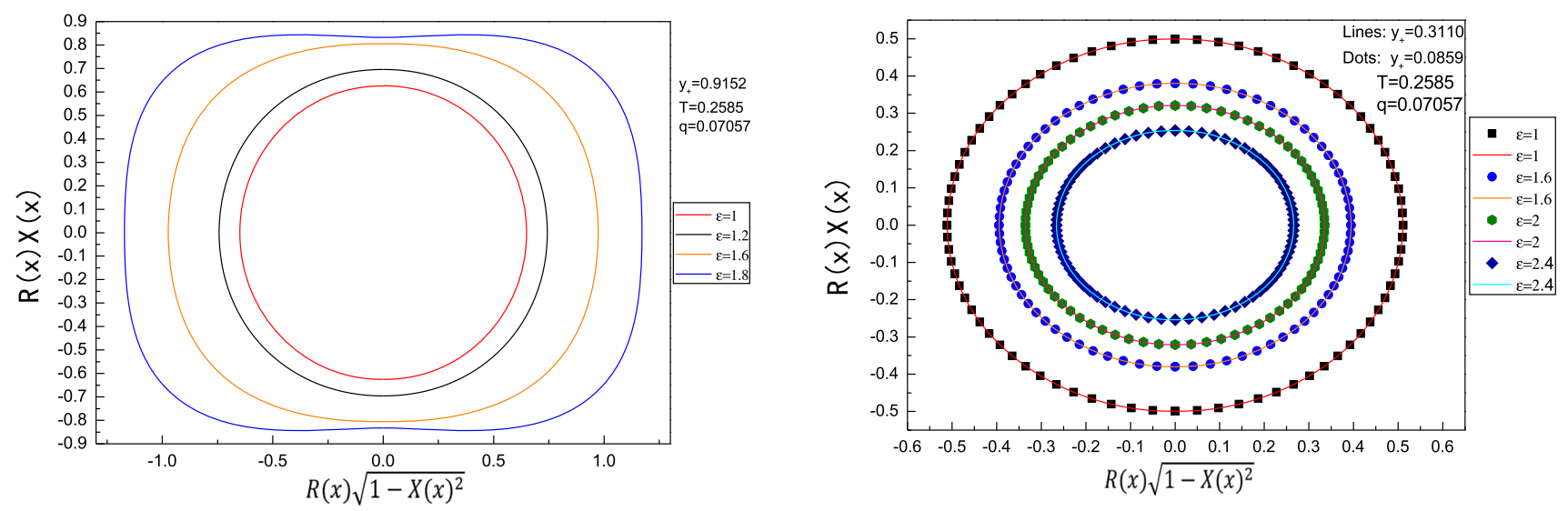

Fig. 4 Hyperbolic embedding of the cross section of three black hole horizons for different values of $\varepsilon$ at $T=0.2585$ and $q=0.07057$. Left: the horizon geometry of large branch for $y_{+}=0.9152$. Right: the horizon geometry of two small branches for $y_{+}=0.3110$ (lines) and $y_{+}=0.0859$ (dots)

Fig. 5 Hyperbolic embedding of the cross section of the large black hole horizon for different values of charge $q$ with $y_{+}=1.5$ and $\varepsilon=1.6$

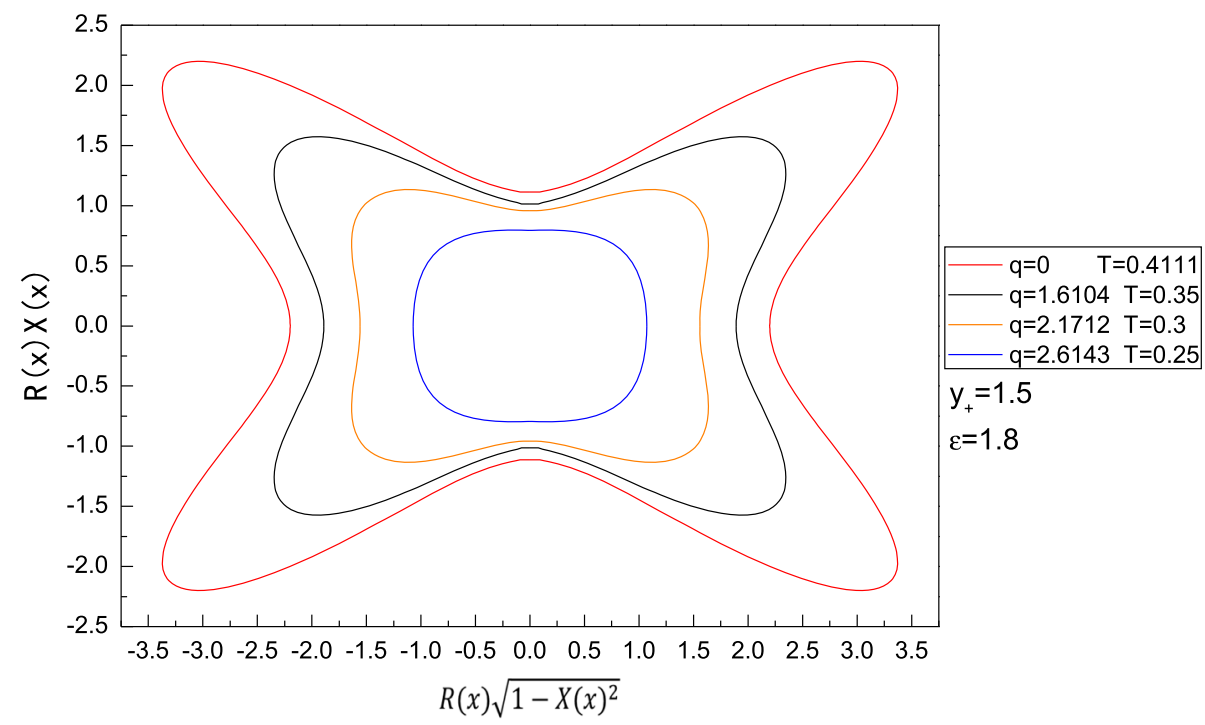

tion increases as $\varepsilon$ increases, which is similar to the results in Fig. 3. In the right panel, we show the result of two small branches for $y_{+}=0.3110$ (lines) and $y_{+}=0.0859$ (dots). It is different from the left panel that the size of the deformation of horizon is a decreasing function of boundary rotation parameters $\varepsilon$. For the two small branches with different horizon radii, the horizon radius of the bigger one is nearly four times as that of the smaller one, but it is interesting to find that the two small branches have same embedding graphs of horizon geometry.

To show the effect of charge $q$ on the deformation of horizon, we give hyperbolic embedding of the cross section of the large black hole horizon for different values of charge $q$ with $y_{+}=1.5$ and $\varepsilon=1.6$ in Fig. 5. Due to the existence of relation (3.6), the temperature decreases with the increasing of charge $q$ for a fixed horizon radius. From outer to inner, the red, black, orange and blue lines represent $q=0,1.6104$, 2.1712 and 2.6143 respectively. The deformation of horizon becomes smaller as the charge $q$ increases.

\subsection{Entropy}

In this subsection, we will discuss the entropy of deforming charged black hole. The formula of entropy of black hole is written as

$S=\frac{A}{4 G_{N}}=\frac{2 \pi y_{+}^{2} L^{2}}{G_{N}} \int_{0}^{1} d x \frac{1-x^{2}}{\sqrt{2-x^{2}}} \sqrt{U_{3}(x, 0) U_{5}(x, 0)}$.

In Fig. 6, we show the entropy against boundary rotation parameter $\varepsilon$ with $T=0.42$ and $q=0.07057$. The entropy of large black hole with $y_{+}=1.5438$ is shown in the left panel, while in the right panel, two small branches with $y_{+}=$ 0.1773 and $y_{+}=0.0992$ are represented by red line and black dots respectively. For the large black hole, the entropy is an increasing function of boundary rotation parameter $\varepsilon$ and approaches infinity as $\varepsilon \rightarrow 2$. Similar to the case without charge in [15], the Kretschmann curvature of large branch goes to infinity when $\varepsilon \rightarrow 2$, which means the formation of 


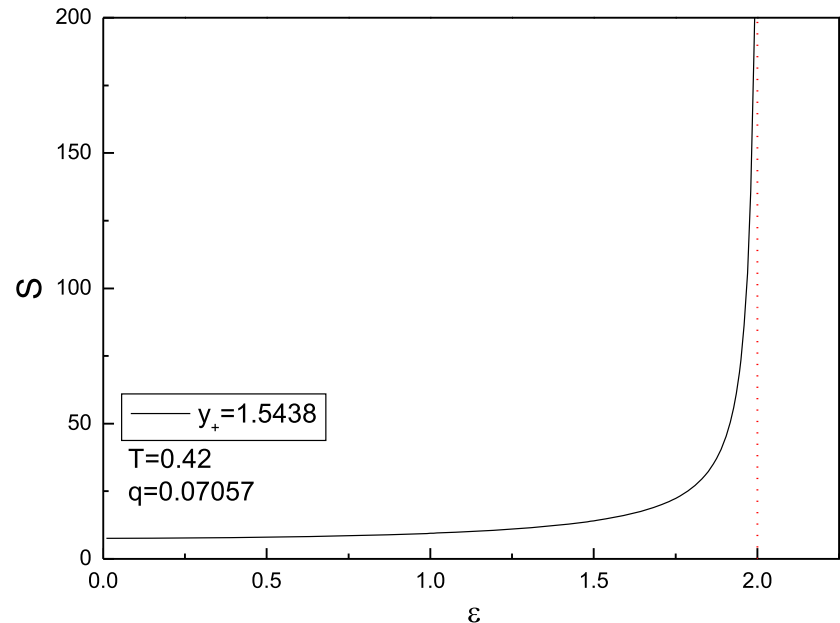

Fig. 6 The entropy as functions of boundary rotation parameter $\varepsilon$ for the temperature $T=0.42$ and the charge $q=0.07057$. Left: the entropy against boundary rotation parameter $\varepsilon$ for the large branch of black hole solutions $y_{+}=1.5438$. Right: the entropy against boundary rotation

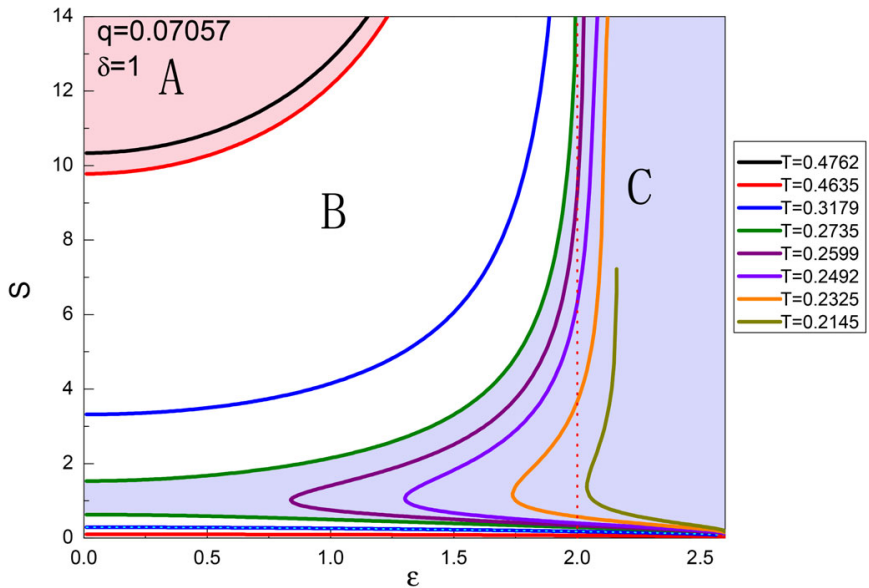

Fig. 7 The entropy as functions of boundary rotation parameter $\varepsilon$ for different values of temperature $T$ with $\delta=1$. Left: for $q=0.07057$, local maximum and minimum of temperature $T$ are equal to 0.4635 and 0.2735 represented by red and green lines respectively. The two

singularity. So the scalar invariants such as entropy would also goes to infinite when $\varepsilon \rightarrow 2$ and we could not find charged deforming black hole solutions when $\varepsilon>2$. As for two small branches with a fixed temperature, the entropy decreases with the increase of boundary rotation parameter $\varepsilon$, and there still exist solutions when $\varepsilon>2$. Furthermore, we also find another family of small black hole solutions, and in these solutions, the entropy increases with the increase of $\varepsilon$.

To obtain the complete phase diagram of entropy for $\delta=$ 1 , we investigate the whole region of temperature in terms of entropy. The entropy as functions of boundary rotation parameter $\varepsilon$ for different values of temperature $T$ with $\delta=1$

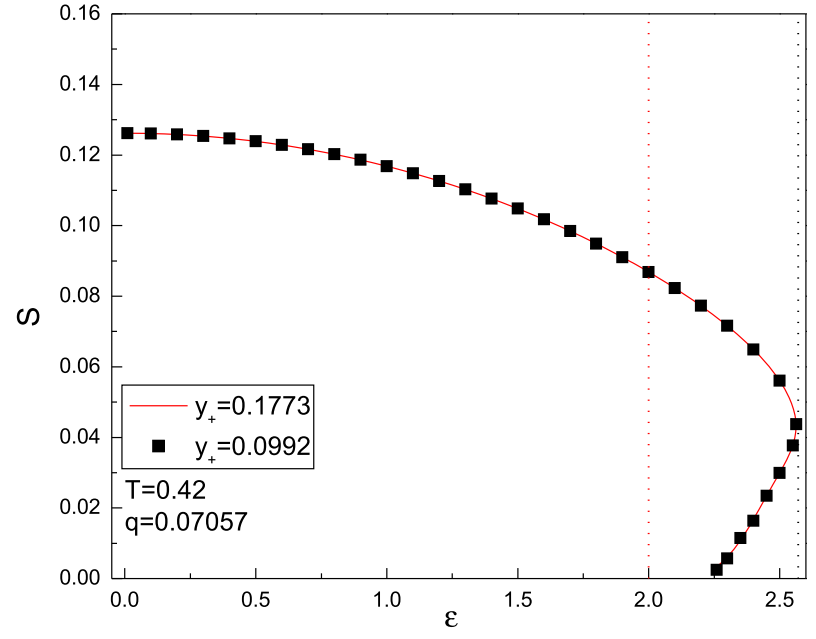

parameter $\varepsilon$ for two small branches of black hole $y_{+}=0.1773$ (red line) and $y_{+}=0.0992$ (black dots). The vertical red and black dot lines represent $\varepsilon=2$ and $\varepsilon=2.57$ respectively



extremums divide the phase diagram of entropy into three regions. Right: for $q=1.7068$, there is always one solution without extremum of temperature $T$. The vertical red dot lines represent $\varepsilon=2$

is shown in Fig. 7. In the left panel, when we fix $q=0.07057$, there are two local extremums $T_{\max }=0.4635$ and $T_{\min }=$ 0.2735 , the entropy of which are represented by red and green lines respectively. The two extremums divide the temperature into three regions:

- Region A with $T>T_{\max }$ : There is only one value of horizon for a fixed temperature and the entropy increases with the increasing of boundary rotation parameter $\varepsilon$. The region $\mathrm{A}$ is indicated by the red area.

- Region B with $T_{\min }<T<T_{\max }$ : There are three values of horizon for a fixed temperature. For the large branch 
of black hole, the entropy increases with $\varepsilon$. Although these two small branches have different black hole horizon radii, they have same entropy which is a decreasing function of boundary rotation parameter $\varepsilon$.

- Region $\mathrm{C}$ with $T<T_{\min }$ : There is only one horizon, but we could find two branches of entropy. The entropy increases with rotation parameter $\varepsilon$ at one branch, while it is a decreasing function of $\varepsilon$ in another branch. It is notable that when $T \leq T_{R N} \approx 0.2599$, the two branches of entropy for one temperature join up. The region $\mathrm{C}$ is indicated by the blue area.

In the right panel of Fig. 7, we fix $q=1.7068>\frac{1}{6}$. There only exists one value of horizon for any temperature, but we could obtain two branches of entropy. The entropy increases with rotation parameter $\varepsilon$ at one branch, while it is a decreasing function of $\varepsilon$ in another branch. It is notable that when $T \leq T_{R N}$, the two branches of entropy could connect, which is similar to the region $\mathrm{C}$ in left panel.

Similar to Sect. 4.1, we adjust $\delta<1$ to get three values of horizon and study the entropy for a fixed low temperature. In Fig. 8, we exhibit entropy as functions of boundary rotation parameter $\varepsilon$ for different values of temperature $T$ at $q=0.07057$. The minimal temperature $T=0.2735$ for $\delta=1$ is represented by black lines. For a fixed temperature $T \leq T_{\min } \approx 0.2599$, the large branch join up with two small branches, which form a set of lines. At each set, the line showing that entropy increases with the increasing of $\varepsilon$ describes large branch and corresponding solid line and dot line describe two small branches. From left to right, these sets of lines indicate $T=0.2599,0.2492,0.2325,0.2257$ and 0.2104 , respectively. Similar to above results in the right panel of Fig. 6, the two small branches have same entropy. When the temperature $T$ is lower than $T_{\min }$, the large branches also have solutions with $\varepsilon>2$. The entropy become infinity when $\varepsilon$ approaches to a maximum value of solutions.

\subsection{Stability}

In this section, we study the stability of deforming charged black hole solutions. Following the method provided in $[15,33,34]$, we consider a free, massless and neutral scalar field perturbation to background and solve the Klein-Gordon equation

$\square \Phi=0$,

and we could decompose the scalar field as the following standard form

$\Phi=\hat{\Phi}_{\omega, m}(x, y) e^{-i \omega t+i m \phi}$,

where the constant $\omega$ is the frequency of the complex scalar field and $m$ is the azimuthal harmonic index. Considering

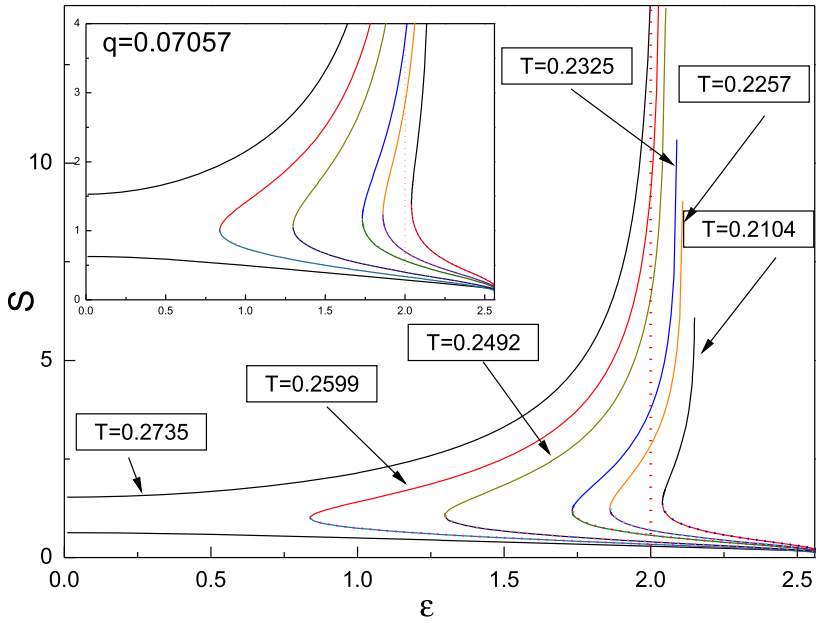

Fig. 8 The entropy as functions of boundary rotation parameter $\varepsilon$ for $T \leq T_{\min }=0.2735$ with $q=0.07057$. The black lines indicates $T=T_{\min }$ and the red vertical dash line indicates the $\varepsilon=2$



Fig. 9 The real part of frequencies $\omega$ against the rotation parameter $\varepsilon$ of two small branches for different value of angular quantum number $m$ at $T=0.42$ and $q=0.07057$. The black horizontal line represents $\operatorname{Re} \omega=0$. The black vertical line represents $\varepsilon=2$

the ingoing Eddington-Finkelstein coordinates [35,36], the scalar field with the ansätze of the black hole metric (3.1) could be decomposed into

$$
\begin{aligned}
\Phi(t, x, y, \phi)= & e^{-i \omega t} e^{i \omega \phi} y^{-i \frac{2 \omega y_{+}}{1+3 y_{+}^{2}}} \\
& \left(1-y^{2}\right)^{3}\left(1-x^{2}\right)^{|m|} \psi(x, y),
\end{aligned}
$$

where the powers of $x$ and $y$ are chosen to make function $\psi(x, y)$ regular at the origin. The boundary conditions are imposed as follow:

$$
\left\{\begin{array}{l}
\partial_{x} \psi(x, y)=0, \quad x= \pm 1, \\
\partial_{y} \psi(x, y)=0, \quad y=0, \\
-2 i y_{+} \omega \psi(x, y)+\left(1+3 y_{+}^{2}\right) \partial_{y} \psi(x, y)=0, \quad y=1 .
\end{array}\right.
$$


In Fig. 9, we give the real part of quasinormal frequencies $\omega$ against the rotation parameter $\varepsilon$ of two small branches for different values of angular quantum number $m$. From top to bottom, these dot lines represent $m=5,8,10,13$ and 16 respectively. Similar to the above results of horizon geometric and entropy, these two small branches have equal quasinormal frequencies though the horizon radius of the bigger one is nearly twice as that of the smaller one. The real part of frequencies $R e \omega$ is always positive when $m<13$. When $m \geq 13, \operatorname{Re} \omega$ would appear a negative value with the increase of rotation parameter $\varepsilon$, which means we could obtain a stable deforming charged black hole solution with scalar condensation.

\section{Black hole solutions with $\mu=\frac{2 q}{y_{+}}$}

In the right panel of Fig. 6 in Sec. 4, the entropy of two small branches are equal to about 0.1275 when $\varepsilon=0$. For the standard RN-AdS black hole, the entropy is equal to $\pi r_{+}^{2}$, so the entropy of two small branches should be equal to 0.0988 and 0.0309 respectively. The reason of the inconsistency is that we fix chemical potential $\mu$ in our calculation. In this section, we would choose $\mu=\frac{2 q}{y_{+}}$according to different values of horizon radii, which ensures the deforming charged AdS black hole solutions can reduce to standard RN-AdS black holes when $\varepsilon=0$.

We would show the numerical results of two small branches for $T=0.42$ and $q=0.07057$. In Fig. 10, we show the entropy against boundary rotation parameter $\varepsilon$ and



Fig. 10 Numerical results of two small branches for $T=0.42$ and $q=0.07057$ when choosing $\mu=\frac{2 q}{y_{+}}$. The values of $\mu$ are equal to 0.796 and 1.423 for $y_{+}=0.1773$ and $y_{+}=1.423$ respectively. Left: the entropy of two small branches as functions hyperbolic embedding of the cross section of horizon for two small branches. The real part of frequencies $\omega$ against the rotation parameter $\varepsilon$ of two small branches is shown in Fig. 11. In each figure, the red line represents $y_{+}=0.0992$ and black dots represent $y_{+}=0.1773$, and the corresponding values of $\mu$ are equal to 1.423 and 0.796 respectively, which ensure that the solutions can reduce to standard RNAdS black holes. Form the left panel of Fig. 10, we could find that the entropy of two small branches are equal to 0.099 and

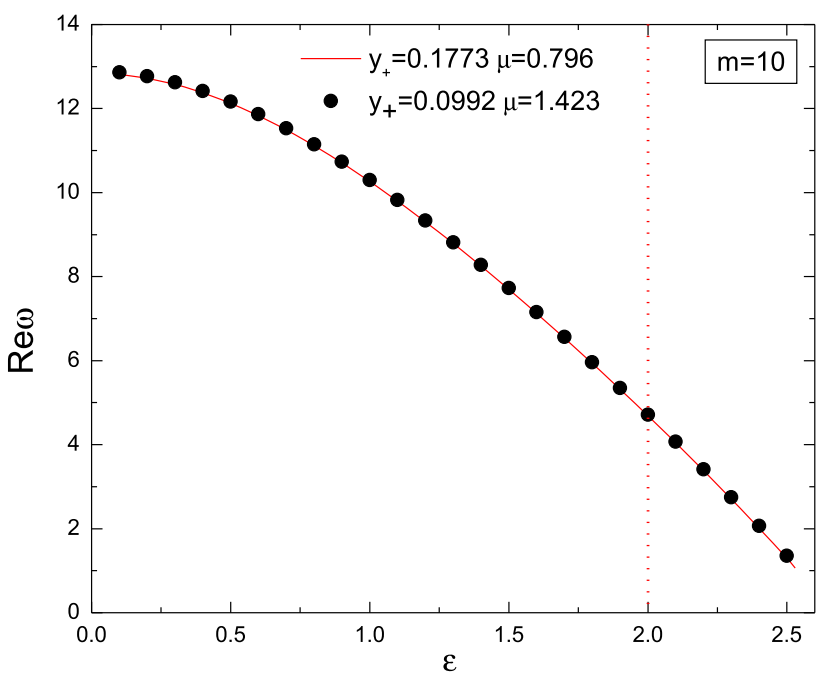

Fig. 11 The real part of frequencies $\omega$ against the rotation parameter $\varepsilon$ of two small branches when choosing $\mu=\frac{2 q}{y_{+}}$. The values of $\mu$ are equal to 0.796 and 1.423 for $y_{+}=0.1773$ and $y_{+}=1.423$ respectively. The red vertical line represents $\varepsilon=2$

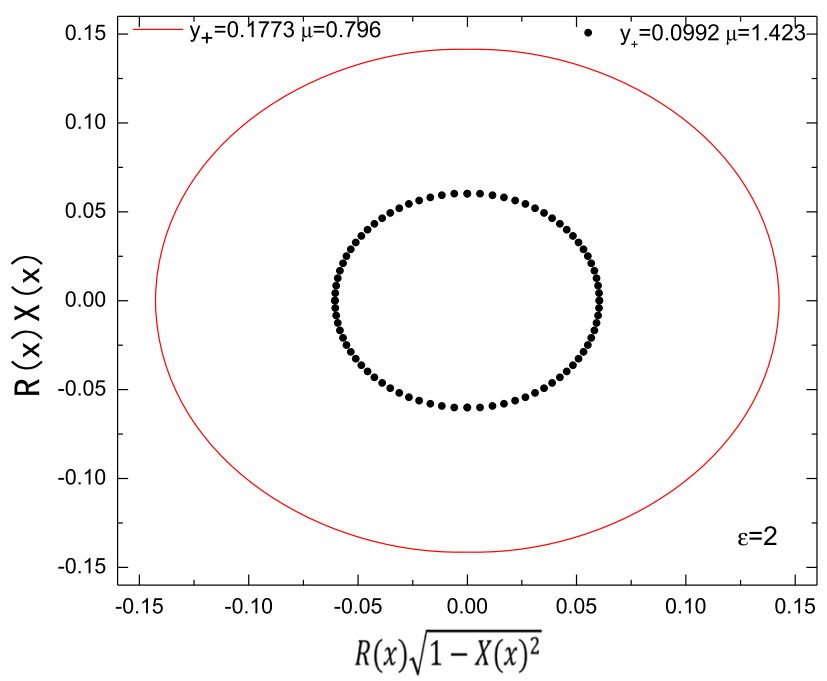

of boundary rotation parameter $\varepsilon$. The vertical red and black dot lines represent $\varepsilon=2$ and $\varepsilon=2.57$ respectively. Right: hyperbolic embedding of the cross section of two small black hole horizons 
0.031 when the boundary rotation $\varepsilon \rightarrow 0$, which is consistent with the result of standard $\mathrm{RN}-\mathrm{AdS}$ black hole. It is notable that the branch with larger horizon radius still has the second family of solutions, while the entropy of the branch with smaller horizon radius decreases to 0 as the increasing of $\varepsilon$. For the horizon geometry shown in the right panel of Fig. 10, it is clear that the branch with larger horizon radius is in the outer of smaller branch. In Fig. 11, we find that these two small branches have equal quasinormal frequencies, which is similar to the results given in Fig. 9. Since quasinormal frequency is a physical property of black hole, it does not depend on different values of $\mu$.

\section{Conclusions and outlook}

In this paper, we studied the conformal boundary of fourdimensional static asymptotically AdS solutions in EinsteinMaxwell gravity and constructed solutions of deforming charged AdS black hole. In contrast with the cases without charge, the charge $q$ could influence the extremums of temperature $T$ which divide the range of temperature into different regions according to the value of the charge $q$. The number of horizons depends on the different regions of temperature $T$. Moreover, there exists no horizon when $T<T_{\min }$ for $q=0$, but there is at least one value of horizon for a fixed temperature when we take the charge $q \neq 0$.

We also investigated physical properties for charged deforming AdS black holes, including the deformation of horizon, entropy and stability.

- Deformation of horizon: In the region with three values of horizon for a fixed temperature, the deformation of horizon for large branch increases with the increasing of boundary rotation parameter $\varepsilon$, while that of small branches is a decreasing function of $\varepsilon$, which shows very similar results to the cases without charge. We also studied how the horizon deforms against the charge $q$ and found that the deformation of horizon became smaller as the charge $q$ increases.

- Entropy: In the region with three values of horizon for a fixed temperature, with the increase of $\varepsilon$, the entropy of large branches increases, while that of small branches decreases. There also exist another set of unstable solutions of small branches, where the entropy increases with the increasing of $\varepsilon$. The entropy of large branch and small branches for a fixed temperature join up when temperature $T$ is lower than $T_{R N}$. It is worth noting that in the region with one value of horizon for a fixed temperature, we could find two families of solutions with same horizon radius, and they have different properties of entropy when the temperature $T<T_{R N}$.
- Stability: We have studied the stability of scalar fields in the background of deforming charged AdS black holes, and found that when angular quantum number $m \geq 13$, the real part of frequencies begins to appear negative values, which means scalar condensation.

The most interesting finding in our research is that when we fix the value of chemical potential $\mu$, in the region with three values of horizon at one temperature, the two small branches for a fixed temperature have same numerical results, including deformation of horizon, entropy and stability though their horizon radii might vary many times. When we take $\mu=\frac{2 q}{y_{+}}$to require the deforming charged AdS black hole solutions to reduce to standard RN-AdS black holes with boundary rotation parameter $\varepsilon=0$, the black holes with different horizon radii have different horizon geometry and entropy.

It is still unclear why these two small branches with different horizon radii for a fixed temperature have the same horizon geometry and entropy. It is worth studying in our future work. At present, we have studied the horizon geometry, entropy and stability of charged AdS black hole with differential rotation boundary. But the angular momentum, energy densities and thermodynamic relation of deforming charged black hole have not been studied, and we hope to investigate these in our future work. Besides, we are planning to study the deforming charged black holes in $f(R)$ gravity and nonlinear electrodynamics.

Acknowledgements We would like to thank Yu-Xiao Liu and Jie Yang for helpful discussion. We also thank the anonymous referees for their valuable comments which helped to improve the manuscript. Some computations were performed on the Shared Memory system at Institute of Computational Physics and Complex Systems in Lanzhou University. This work was supported by the Fundamental Research Funds for the Central Universities (Grants no. lzujbky-2017-182).

Data Availability Statement This manuscript has no associated data or the data will not be deposited. [Author0s' comment: The datasets generated during the current study are available from the corresponding author on reasonable request.]

Open Access This article is licensed under a Creative Commons Attribution 4.0 International License, which permits use, sharing, adaptation, distribution and reproduction in any medium or format, as long as you give appropriate credit to the original author(s) and the source, provide a link to the Creative Commons licence, and indicate if changes were made. The images or other third party material in this article are included in the article's Creative Commons licence, unless indicated otherwise in a credit line to the material. If material is not included in the article's Creative Commons licence and your intended use is not permitted by statutory regulation or exceeds the permitted use, you will need to obtain permission directly from the copyright holder. To view a copy of this licence, visit http://creativecomm ons.org/licenses/by/4.0/.

Funded by SCOAP 3 . 


\section{References}

1. W. Israel, Event horizons in static vacuum space-times. Phys. Rev. 164, 1776 (1967)

2. R. Ruffini, J.A. Wheeler, Introducing the black hole. Phys. Today 24, 30 (1971)

3. P.T. Chrusciel, J. Lopes Costa, M. Heusler, Stationary black holes: uniqueness and beyond. Living Rev. Rel. 15, 7 (2012). arXiv:1205.6112 [gr-qc]

4. B. Carter, C. De Witt, B.S. De Witt, in Proceedings of 1972 Session of Ecole dEte De Physique Theorique (Gordon and Breach, New York, 1973)

5. J.M. Maldacena, The large $N$ limit of superconformal field theories and supergravity. Int. J. Theor. Phys. 38, 1113 (1999)

6. E. Witten, Anti-de Sitter space and holography. Adv. Theor. Math. Phys. 2, 253 (1998). arXiv:hep-th/9802150

7. O. Aharony, S.S. Gubser, J.M. Maldacena, H. Ooguri, Y. Oz, Large $\mathrm{N}$ field theories, string theory and gravity. Phys. Rep. 323, 183 (2000). arXiv:hep-th/9905111

8. Y. Chen, Y.K. Lim, E. Teo, Deformed hyperbolic black holes. Phys. Rev. D 92(4), 044058 (2015). https://doi.org/10.1103/PhysRevD. 92.044058. arXiv:1507.02416 [gr-qc]

9. Levi-Civita, T. ds2 einsteiniani in campi newtoniani. VII. Il sottocaso B2: soluzioni oblique (in Italian). Rend. Acc. Lincei 27:343 (1918)

10. H. Weyl, Zur Gravitationstheorie (in German). Ann. Phys. 54, 117 (1917)

11. J.F. Plebanski, M. Demianski, Rotating, charged, and uniformly accelerating mass in general relativity. Ann. Phys. 98, 98 (1976). https://doi.org/10.1016/0003-4916(76)90240-2

12. A. Gnecchi, K. Hristov, D. Klemm, C. Toldo, O. Vaughan, Rotating black holes in $4 \mathrm{~d}$ gauged supergravity. JHEP 1401, 127 (2014). https://doi.org/10.1007/JHEP01(2014)127. arXiv:1311.1795 [hep-th]

13. D. Klemm, Four-dimensional black holes with unusual horizons. Phys. Rev. D 89(8), 084007 (2014). https://doi.org/10.1103/ PhysRevD.89.084007. arXiv:1401.3107 [hep-th]

14. Y. Chen, E. Teo, Black holes with bottle-shaped horizons. Phys. Rev. D 93(12), 124028 (2016). https://doi.org/10.1103/PhysRevD. 93.124028. arXiv:1604.07527 [gr-qc]

15. J. Markeviit, J.E. Santos, Stirring a black hole. JHEP 1802, 060 (2018). https://doi.org/10.1007/JHEP02(2018)060. arXiv:1712.07648 [hep-th]

16. S.R. Green, S. Hollands, A. Ishibashi, R.M. Wald, Superradiant instabilities of asymptotically anti-de Sitter black holes. Class. Quantum Gravity 33(12), 125022 (2016). https://doi.org/10.1088/ 0264-9381/33/12/125022. arXiv:1512.02644 [gr-qc]

17. S. Sun, T.T. Hu, H.B. Li, Y.Q. Wang, Deforming black holes with odd multipolar differential rotation boundary. Phys. Rev. D 100(8), 084063 (2019). https://doi.org/10.1103/PhysRevD.100. 084063. arXiv:1906.06183 [hep-th]

18. H.B. Li, T.T. Hu, B.S. Song, S. Sun, Y.Q. Wang, Deforming black holes with even multipolar differential rotation boundary. JHEP 1906, 126 (2019). https://doi.org/10.1007/JHEP06(2019)126. arXiv:1903.11967 [hep-th]

19. T. Crisford, G.T. Horowitz, J.E. Santos, Attempts at vacuum counterexamples to cosmic censorship in AdS. JHEP 1902, 092 (2019). https://doi.org/10.1007/JHEP02(2019)092. arXiv:1805.06469 [hep-th]

20. G.T. Horowitz, J.E. Santos, C. Toldo, Deforming black holes in AdS. JHEP 1811, 146 (2018). https://doi.org/10.1007/ JHEP11(2018)146. arXiv:1809.04081 [hep-th]
21. J.L. Blzquez-Salcedo, J. Kunz, F. Navarro-Lrida, E. Radu, New black holes in $\mathrm{D}=5$ minimal gauged supergravity: deformed boundaries and frozen horizons. Phys. Rev. D 97(8), 081502 (2018). https://doi.org/10.1103/PhysRevD.97. 081502. arXiv: 1711.08292 [gr-qc]

22. D. Cassani, L. Papini, Squashing the boundary of supersymmetric $\mathrm{AdS}_{5}$ black holes. JHEP 1812, 037 (2018). https://doi.org/10.1007/ JHEP12(2018)037. arXiv:1809.02149 [hep-th]

23. A. Bombini, L. Papini, General supersymmetric $\operatorname{AdS}_{5}$ black holes with squashed boundary. Eur. Phys. J. C 79(6), 515 (2019). https:// doi.org/10.1140/epjc/s10052-019-7015-x. arXiv:1903.00021 [hep-th]

24. M. Headrick, S. Kitchen, T. Wiseman, A new approach to static numerical relativity, and its application to Kaluza-Klein black holes. Class. Quantum Gravity 27, 035002 (2010). https://doi.org/ 10.1088/0264-9381/27/3/035002. arXiv:0905.1822 [gr-qc]

25. T. Wiseman, Numerical construction of static and stationary black holes, in Black holes in higher dimensions, ed. by G. Horowitz (Cambridge University Press, Cambridge, 2012)

26. O.J.C. Dias, J.E. Santos, B. Way, Numerical methods for finding stationary gravitational solutions. Class. Quantum Gravity 33(13), 133001 (2016). https://doi.org/10.1088/0264-9381/33/13/ 133001. arXiv:1510.02804 [hep-th]

27. L. Flamm, Beitrage zur Einsteinischen Gravitationtheorie. Phys. Z. 17, 448-454 (1916). (In particular p. 450)

28. L. Smarr, Surface geometry of charged rotating black holes. Phys. Rev. D 7, 289 (1973)

29. A. Friedman, Isometric embeddings of Riemannian manifolds into Euclidean spaces. Rev. Mod. Phys. 37, 201 (1965)

30. J. Rosen, Embedding various relativistic Riemannian spaces in Pseudo-Euclidean spaces. Rev. Mod. Phys. 37, 204 (1965)

31. Goenner, H. Local isometric embedding of Riemannian manifolds and Einsteins theory of gravitation, in General Relativity and Gravitation: One Hundred Years After the Birth of Einstein, ed by A. Held, vol. 1 (Plenum Press, New York, 1980)

32. G.W. Gibbons, C.A.R. Herdeiro, C. Rebelo, Global embedding of the Kerr black hole event horizon into hyperbolic 3-space. Phys. Rev. D 80, 044014 (2009). https://doi.org/10.1103/PhysRevD.80. 044014. arXiv:0906.2768 [gr-qc]

33. O.J.C. Dias, J.E. Santos, Boundary conditions for Kerr-AdS perturbations. JHEP 1310, 156 (2013). https://doi.org/10.1007/ JHEP10(2013)156. arXiv:1302.1580 [hep-th]

34. V. Cardoso, O.J.C. Dias, G.S. Hartnett, L. Lehner, J.E. Santos, Holographic thermalization, quasinormal modes and superradiance in Kerr-AdS. JHEP 1404, 183 (2014). https://doi.org/10.1007/ JHEP04(2014)183. arXiv:1312.5323 [hep-th]

35. G.T. Horowitz, V.E. Hubeny, Quasinormal modes of AdS black holes and the approach to thermal equilibrium. Phys. Rev. D 62, 024027 (2000). https://doi.org/10.1103/PhysRevD.62.024027. arXiv:hep-th/9909056

36. E. Berti, V. Cardoso, A.O. Starinets, Quasinormal modes of black holes and black branes. Class. Quantum Gravity 26, 163001 (2009). https://doi.org/10.1088/0264-9381/26/16/ 163001. arXiv:0905.2975 [gr-qc] 